\title{
Massless limit of vector multiplets in supergravity
}

\author{
S. Deser \\ Department of Physics, Brandeis University, Waltham, Massachusetts 02254
}

(Received 3 December 1979)

\begin{abstract}
The massless limit of a vector multiplet coupled to supergravity reduces smoothly to a sum of separately coupled massless vector and scalar multiplets. Suitable field-dependent chiral rotations of the fermions lead to a particularly symmetric form for the latter.
\end{abstract}

Two recent papers $\mathrm{s}^{1,2}$ have derived the coupling of a massive vector supermultiplet to supergravity. In this note, we show that-contrary to the statements in Ref. 1 - the $m=0$ limit is smooth and leads to a system of separately coupled massless vector and scalar multiplets, ${ }^{3}$ in accord with a naive degree-of-freedom count. The resulting system, incidentally, provides an example of supergravity in interaction with two separate supermultiplets.

Since scalars and spinors have smooth massless limits, the only delicate point lies in the spin-1 sector. But it is well known that there is no problem for the latter either if due care is taken to redefine the third, longitudinal, degree of freedom. $^{4,5}$ Indeed, it is also known that although it decouples from electric charge as $m \rightarrow 0$, it does not decouple from gravity. ${ }^{5}$ We shall show, in effect, that this longitudinal field remains coupled to full supergravity and becomes the scalar component of the massless scalar multiplet.

For convenience, we shall use the notation and results of Ref. 1. Let us first consider flat space. Here,

$$
\begin{aligned}
L_{V}(m) & =L_{K}+L_{m} \\
& =\left[-\frac{1}{4} F_{\mu \nu}{ }^{2}+\frac{1}{2} i \bar{\chi}_{i} \not \chi_{i}+\frac{1}{2}\left(\partial_{\mu} \varphi\right)^{2}\right] \\
& +\left(\frac{1}{2} m^{2} A_{\mu}{ }^{2}-m \bar{\chi}_{i} \chi_{i}-\frac{1}{2} m^{2} \varphi^{2}\right)
\end{aligned}
$$

is the massive vector multiplet's action in terms of a Proca field $A_{\mu}$, two Majorana spinors $\chi_{i}$, and a pseudoscalar $\varphi$, invariant under

$$
\begin{aligned}
& \sqrt{2} \delta A_{\mu}=i \bar{\epsilon} \gamma_{\mu} \chi_{1}+m^{-1} \partial_{\mu} \bar{\epsilon} \chi_{2}, \quad \sqrt{2} \delta \varphi=i \bar{\epsilon} \gamma_{5} \chi_{2}, \\
& \sqrt{2} \delta \chi_{1}=\left(\sigma \cdot F-i m \gamma_{5} \varphi\right) \epsilon, \quad \sqrt{2} \delta \chi_{2}=-\left(i m \gamma \cdot A+\gamma_{5} \not \partial \varphi\right) \epsilon .
\end{aligned}
$$

We now assert that the stueckelberg redefinition

$$
A_{\mu}=B_{\mu}^{T}+m^{-1} \partial_{\mu} \theta
$$

permits one to pass to the naive $m \rightarrow 0$ limit, i.e., that there are no hidden singular parts after it is inserted in the action. If this is the case, the Lagrangian (1) becomes simply

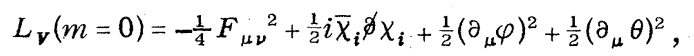

while the corresponding transformations (2) limit to

$$
\begin{aligned}
& \sqrt{2} \delta B_{\mu}=i \bar{\epsilon} \gamma_{\mu} \chi_{1}, \quad \sqrt{2} \delta \chi_{1}=\sigma \cdot F \epsilon, \\
& \sqrt{2} \delta \varphi=i \bar{\epsilon} \gamma_{5} \chi_{2}, \quad \sqrt{2} \delta \theta=\bar{\epsilon} \chi_{2}, \quad \sqrt{2} \delta \chi_{2}=\not\left(\gamma_{5} \varphi-i \theta\right) \epsilon,
\end{aligned}
$$

which are those of two uncoupled multiplets, even though there is an apparently common $\epsilon$.

We shall not take the space here to justify the decomposition (3) in detail; there is lengthy literature ${ }^{4-6}$ on the limit to massless electrodynamics. Briefly, as is emphasized in Refs. 4 and 5 the decomposition [equivalent to (3)]

$$
A_{i}=A_{i}^{T}+m^{-1} \partial_{i} \theta, \quad A_{0}=m^{-1} \partial_{0} \theta
$$

permits one to limit naively: The resulting theory will be the usual electrodynamics with coupling (Ref. 7) $\int j^{\mu} B_{\mu}$, together with a scalar field $\theta$, which is coupled to the current as $m^{-1} \int j^{\mu} \partial_{\mu} \theta$. In the usual situation, $\partial_{\mu} j^{\mu}$ vanishes independent of $m$, and so the longitudinal photon decouples. In the present model, however, things work in precisely the opposite way. There are two types of current couplings: One (from a Noether term) is of the Pauli-moment type $\int M^{\mu \nu} F_{\mu \nu}$, which leads to an identically conserved current and hence sees only the true photons; the other (from both Noether and contact terms) is of "normal" $\int j^{\mu} A_{\mu}$ form, but with $j^{\mu}$ explicitly proportional to $m$. As a result, in the limit its contribution to $\int j^{\mu} B_{\mu}$ vanishes, and only the longitudinal photon remains coupled to it, with the replacement $\int j^{\mu} A_{\mu} \rightarrow$ $\int\left(m^{-1} j^{\mu}\right) \partial_{\mu} \theta$. This is precisely the prescription symbolized by (3). Furthermore, this prescription is manifestly metric independent and consequently can be taken over entirely unchanged to curved space for scalar $\theta$. We may therefore simply transcribe the limit of the supergravity-coupled model directly [now, of course $\epsilon \rightarrow \epsilon(x)]$. The total Lagrangian is the usual supergravity one plus the covariant form of the massless $L_{V}$ of (4), plus Noether and contact terms. The former is quite normal and symmetric in $(\theta, \varphi)$ :

$$
\sqrt{2} L^{(1)}=-e \kappa \bar{\psi}_{\mu}\left[i \sigma \cdot F \gamma^{\mu} \chi_{1}+\gamma^{\nu} \gamma^{\mu} \partial_{\nu}\left(i \gamma_{5} \varphi+\theta\right) \chi_{2}\right] \text {. }
$$

The only unusual part of the contact Lagrangian is 
that arising from a term originally proportional to $m A_{\rho} \varphi \rightarrow\left(\partial_{\rho} \theta\right) \varphi$, namely

$$
\begin{aligned}
& L^{(2)} \sim-\frac{1}{2} \kappa^{2}\left(i \varepsilon^{\mu \nu \lambda \rho} \bar{\psi}_{\mu} \gamma_{\nu} \psi_{\lambda}-e \bar{\chi}_{1} \gamma_{5} \gamma^{\rho} \chi_{1}\right. \\
& \left.+e \bar{\chi}_{2} \gamma_{5} \gamma^{\rho} \chi_{2}\right) \varphi \partial_{\rho} \theta \text {. }
\end{aligned}
$$

Note the $(\theta, \varphi)$ asymmetry here. A similar asymmetry is to be found in the transformation rules for the fermions $\left(\psi_{\mu}, \chi_{i}\right)$. The rules for $\delta \chi_{i}$ are those of (5) together with new $\kappa, \kappa^{2}$ terms. The former are straightforward, the latter are

$$
\sqrt{2} \delta \chi_{1} \sim i \kappa^{2} \varphi \bar{\epsilon} \chi_{2} \gamma_{5} \chi_{1}, \quad \sqrt{2} \delta \chi_{2}=+i \kappa^{2} \varphi \bar{\epsilon} \chi_{2} \gamma_{5} \chi_{2}
$$

with no corresponding $\theta$ parts. The bosonic rules remain as in (5), and of course

$$
\delta e_{\mu a}=-i \kappa \bar{\alpha} \gamma_{a} \psi_{\mu} \text {. }
$$

We now show that all $(\theta, \varphi)$ asymmetries can be removed by suitable field-dependent chiral transformations on the fermions, although this is purely a matter of aesthetics. The hint for this is provided by the identity

$$
\varphi \partial_{\mu} \theta=\frac{1}{2}\left(\varphi \partial_{\mu} \theta-\theta \partial_{\mu} \varphi\right)+\frac{1}{2} \partial_{\mu}(\theta \varphi) \equiv P_{\mu}+\partial_{\mu} \Lambda,
$$

the first term on the right being the usual scalar multiplet's axial-vector current. We next note that the relevant part of $\delta \psi_{\mu}$ may be written as

$$
\begin{aligned}
\delta \psi_{\mu}= & \kappa^{-1}\left[\partial_{\mu}+i \kappa^{2}\left(\partial_{\mu} \Lambda\right) \gamma_{5}\right] \epsilon \\
& -i \frac{\kappa^{2}}{\sqrt{2}} \varphi \bar{\epsilon} \chi_{2} \gamma_{5} \psi_{\mu}+i \kappa P_{\mu} \epsilon .
\end{aligned}
$$

Defining

$$
\begin{aligned}
& \psi_{\mu}^{\prime} \equiv \exp \left(+i \kappa^{2} \Lambda \gamma_{5}\right) \psi_{\mu} \equiv e_{+} \psi_{\mu}, \quad \epsilon^{\prime} \equiv e_{+} \epsilon, \\
& \chi_{1}^{\prime} \equiv e_{+} \chi_{1}, \quad \chi_{2}^{\prime} \equiv e_{-} \chi_{2},
\end{aligned}
$$

and performing the simple algebra, we find that

$$
\begin{aligned}
\delta \psi_{\mu}^{\prime}= & \kappa^{-1} D_{\mu}\left(e, \psi^{\prime}, \chi_{i}^{\prime}\right) \epsilon^{\prime}+i \kappa\left(P_{\mu}+\frac{1}{4} \bar{\chi}_{1}^{\prime} \gamma_{5} \gamma^{\nu} \chi_{1}^{\prime} \gamma_{\nu} \gamma_{\mu}\right) \gamma_{5} \epsilon^{\prime} \\
& +i \frac{\kappa^{2}}{2 \sqrt{2}}\left(i \theta \bar{\epsilon}^{\prime} \gamma_{5} \chi_{2}^{\prime}-\varphi \bar{\epsilon}^{\prime} \chi_{2}^{\prime}\right) \gamma_{5} \psi_{\mu}^{\prime},
\end{aligned}
$$

while the relevant parts of $\delta \chi_{i}$ in (8) become

$$
\begin{aligned}
& \sqrt{2} \delta \chi_{1}^{\prime} \sim-\frac{1}{2} i \kappa^{2}\left(\varphi \bar{\epsilon}^{\prime} \chi_{2}^{\prime}-i \theta \bar{\epsilon}^{\prime} \gamma_{5} \chi_{2}^{\prime}\right) \gamma_{5} \chi_{1}^{\prime}, \\
& \sqrt{2} \delta \chi_{2}^{\prime} \sim+\frac{1}{2} i \kappa^{2}\left(\varphi \bar{\epsilon}^{\prime} \chi_{2}^{\prime}-i \theta \bar{\epsilon}^{\prime} \gamma_{5} \chi_{2}^{\prime}\right) \gamma_{5} \chi_{2}^{\prime},
\end{aligned}
$$

and everything is now symmetric. The other effect of our redefinitions (11) is to symmetrize the contact term (7). For when the kinetic parts of the fermionic actions are reexpressed in terms of the primed variables, the derivatives also act on $e_{ \pm}$. This just transforms the $\varphi \partial_{\rho} \theta$ coefficient in (7) to the axial form $\varphi \partial_{\rho} \theta \rightarrow P_{\rho}$, as is obvious by inspection, remembering the identity (9). Finally, it is also manifest that the redefinitions (11) leave all (nonderivative) fermion bilinears in the action and transformation rules invariant, since bilinears in those which transform cogrediently, $\left(\psi_{\mu}, \epsilon, \chi_{1}\right)$ always involve $\gamma_{\mu}$ or $\gamma_{5} \gamma_{\mu}$, while the oppositely transforming $\chi_{2}$ always enters in the combinations $\bar{\epsilon} \chi_{2}$, etc., with the former.

We conclude then that there is a perfectly clean $m \rightarrow 0$ limit for the supergravity-vector-multiplet system, namely that of two massless multiplets $\left(F_{\mu \nu}, \chi_{1}\right)$ and $\left(\chi_{2}, \varphi, \theta\right)$, separately interacting with gravity in a polynomial way, with straightforward Noether and contact terms (doubtless simplifiable by use of auxilliary fields), and full symmetry between $(\theta, \varphi)$. The corresponding local transformations are likewise appropriate to the reduced system and $(\theta, \varphi)$ are symmetric.

Note added. Our final action and transformation rules also agree with a suitable limit of massless QED coupled to supergravity. ${ }^{8}$ That system consists of a photon and its associated fermion $\lambda$, together with two massless scalar superdoublets $\left(A_{i}, B_{i} ; \chi_{i}\right), i=1,2$. It is consistent there to set one of the multiplets $(i=1)$, as well as the charge $e$ and parity-violating parameter $\zeta$, equal to zero. Renaming $A_{2}=\theta, B_{2}=\phi, \lambda=\chi_{1}$ in Eqs. (6.2) and (6.4) of Ref. 8 gives the present form [our global chiral invariance is also found in (6.6)]. I thank D. Z. Freedman for reminding me of this model.

This work was supported in part by NSF Grant No. PHY-78-09644 A01.
${ }^{1}$ S. Mukhi, Phys. Rev. D 20, 1839 (1979).

${ }^{2}$ A. VanProyen, Nucl. Phys. B162, 376 (1980).

${ }^{3}$ S. Ferrara, D. Z. Freedman, P. van Nieuwenhuizen, P. Breitenlohner, F.Gliozzi, and J.Scherk, Phys.Rev. D 15, 1013 (1977); D. Z. Freedman and J. Schwarz, ibid. $\overline{15}, 1007$ (1977).

${ }^{4}$ D. G. Boulware and W. Gilbert, Phys. Rev. 126, 1563 (1962).

${ }^{5}$ S. Deser, Ann. Inst. Henri Poincaré A16, 79 (1972).

${ }^{6}$ F. Coester, Phys. Rev. 83, 798 (1951); H. Umezawa, Prog. Theor. Phys. 7, $\overline{551}$ (1952); R. J. Glauber, ibid.

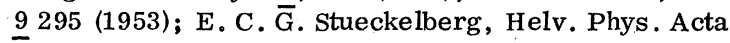

30, 209 (1957).

${ }^{7}$ The apparent paradox that only $B_{i}^{T}$ remains, so that the $\left(B^{L}, B^{0}\right)$ parts of the vector potential are missing, is simply resolved. In the $B$ sector, the action is of the form

$$
I_{\max }\left(B_{i}^{T}\right)+\int \overrightarrow{\mathrm{j}}^{T} \cdot \overrightarrow{\mathrm{B}}^{T}+\frac{1}{2} \int j^{0}\left(\nabla^{-2}\right) j^{0} .
$$

By "inventing" two new variables $\left(B^{L}, B^{0}\right)$, one regains the usual covariant $\int j^{\mu} B_{\mu}$ form.

${ }^{8}$ A. Das, M. Fischler, and M. Rocek, Phys. Rev. D 16, 3427 (1977). 